\title{
Effects of impurity atoms and molecules on the lifetime of antiprotonic helium atoms
}

\author{
E. Widmann, ${ }^{*}$ I. Sugai, and T. Yamazaki \\ Institute for Nuclear Study, University of Tokyo, 3-2-1 Midori-cho, Tanashi, Tokyo 188, Japan \\ R. S. Hayano, M. Iwasaki, S. N. Nakamura, ${ }^{\dagger}$ H. Tamura, T. M. Ito, and A. Kawachi \\ Department of Physics, University of Tokyo, 7-3-1 Hongo, Bunkyo-ku, Tokyo 113, Japan \\ N. Nishida and W. Higemoto \\ Tokyo Institute of Technology, O-Okayama, Meguro-ku, Tokyo 152, Japan \\ Y. Ito \\ Research Center for Nuclear Science and Technology, University of Tokyo, Tokai, Ibaraki 319-11, Japan \\ N. Morita \\ Institute for Molecular Science, Myodaiji, Okazaki 444, Japan \\ F. J. Hartmann, H. Daniel, T. von Egidy, W. Schmid, and J. Hoffmann \\ Physik Department, Technische Universität München, D-85747 Garching, Germany \\ J. Eades \\ CERN, CH-1211 Geneva 23, Switzerland
}

(Received 10 November 1995)

\begin{abstract}
Delayed annihilation time spectra (DATS) of antiprotons in room-temperature helium gas have been studied as a function of the concentration of admixed noble gases $(\mathrm{Ne}, \mathrm{Ar}, \mathrm{Kr}, \mathrm{Xe})$ as well as molecular gases $\left(\mathrm{N}_{2}, \mathrm{O}_{2}, \mathrm{H}_{2}\right)$ at the low-energy antiproton ring at CERN. The DATS were a superposition of two exponential components, one with a lifetime of several $100 \mathrm{~ns}$ and the other with a lifetime 1-3 $\mu$ s. They showed a shorter average lifetime $\left(T_{\mathrm{av}}\right)$ than DATS of pure helium. $\mathrm{Ne}, \mathrm{Ar}$, and $\mathrm{Kr}$ were found to affect $T_{\mathrm{av}}$ only slightly even in concentrations up to $20 \%$, while Xe showed a much stronger influence. In the case of molecular gases, the presence of $\mathrm{N}_{2}$ influenced the DATS much less than $\mathrm{O}_{2}$ and $\mathrm{H}_{2}$, which destroyed the metastability almost completely in concentrations of $100 \mathrm{ppm}$ and less. The decay rate of the slow component of DATS was found to exhibit a linear relation to the number density of the admixture. From this a collisional destruction ("quenching") cross section $\sigma_{q}$ was extracted. No evidence for a delayed annihilation of antiprotons in pure $\mathrm{Ne}, \mathrm{Kr}$, and $\mathrm{Xe}$ was observed.
\end{abstract}

PACS number(s): $36.10 .-\mathrm{k}$

\section{INTRODUCTION}

Delayed annihilation of antiprotons in helium was observed in an experiment by Iwasaki et al. [1]. About $3 \%$ of antiprotons stopped in liquid helium were found to survive with an average lifetime of about $3 \mu \mathrm{s}$. Similar effects had been observed for $K^{-}$[2] and $\pi^{-}$[3] in liquid helium. No evidence was found, however, for delayed annihilation in liquid nitrogen or argon.

This discovery is in contradiction to the common understanding that negatively charged hadrons when stopped in matter are instantly (i.e., within $10^{-12} \mathrm{~s}$ ) absorbed by the nucleus. The observed metastability can be explained by the formation of long-lived states of exotic helium atoms $\bar{p} e^{-} \mathrm{He}^{+}\left(=\bar{p} \mathrm{He}^{+}\right)$, with the antiproton $(\bar{p})$ bound in a state with large principal quantum number $n \approx n_{0}$

\footnotetext{
*Present address: CERN, CH-1211 Geneva 23, Switzerland.

Present address: The Institute of Physical and Chemical Research (RIKEN), Wako, Saitama 351-01, Japan.
}

$=\sqrt{M^{*} / m_{e}}\left(M^{*}\right.$ being the reduced mass and $m_{e}$ the mass of the electron), and large orbital angular momentum $l \approx n_{0}-1$. The level spacing of these "circular orbits" is $\approx 2 \mathrm{eV}$, while the ionization energy is $\approx 25 \mathrm{eV}$ for He. Energy conservation then makes fast Auger transitions (i.e., those with a small change of $l$ ) impossible. Under these conditions radiative transitions can occur even though they are very slow. Additional deexcitation processes such as Stark mixing that are normally dominant for exotic atoms in dense media are suppressed due to the presence of the electron and the resulting neutrality of the atom.

This model was suggested by Condo [4] to explain the anomalous free-decay fractions of $\pi^{-}$and $K^{-}$observed in liquid-helium bubble chambers. It was further developed and extended to the case of antiprotons by Russell [5] (for historical accounts, see [6]). Recently, several authors have calculated the energy levels and radiative lifetimes of these levels using, respectively, a configuration mixing approach [7], a molecular approach [8-10], the large-space variational method [11], and a nonadiabatic coupled rearrangement channel variational calculation [12]. All calculations yield 


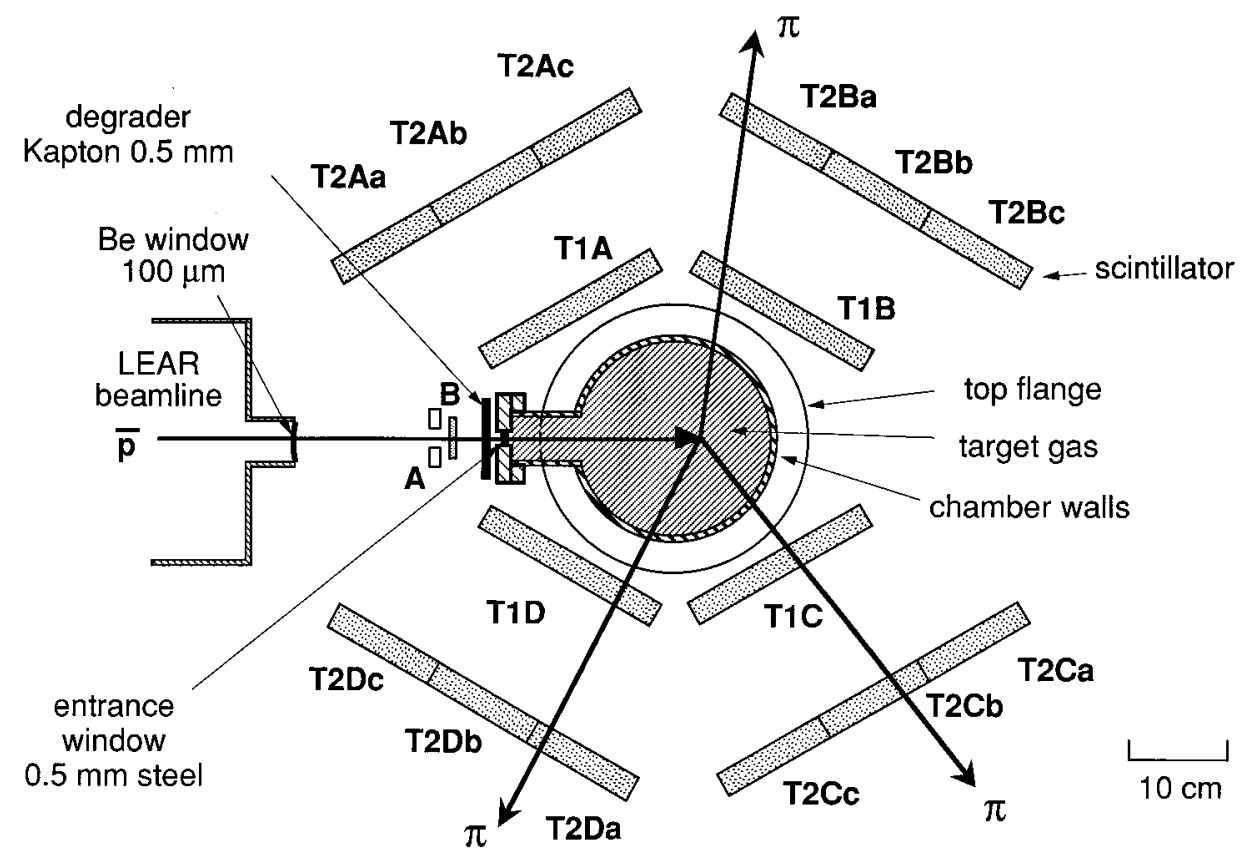

FIG. 1. Setup to measure delayed annihilation time spectra of antiprotons in helium (top view). The $B$ and $A$ counters have been drawn on an enlarged scale. In the center the target-gas chamber is shown.

similar level energies and radiative lifetimes of about 1-2 $\mu \mathrm{s}$. To get the total decay rates the Auger rates of course need to be known as well as the radiative ones. A recent calculation of Ohtsuki and co-workers $[13,14]$ shows that only those states that can proceed to lower-lying ionized states of $\bar{p} \mathrm{He}^{2+}$ via $\Delta l \leqslant 3$ Auger emission are "short lived" ( $\tau \leqslant 10 \mathrm{~ns}$ ), the overall lifetimes of all other states being dominated by radiative deexcitation. The $(n, l)$ energylevel diagram is thus divided into a metastable and a shortlived zone, as shown in $[7,15]$. Recent laser resonance experiments [16-18] have confirmed this picture of the overall structure of the $\bar{p} \mathrm{He}^{+}$atom.

Experimentally observed delayed annihilation time spectra (DATS) depend on the distribution of the initial population over $(n, l)$ as well as the possible quenching of the metastable $\bar{p} \mathrm{He}^{+}$atoms after their formation by atoms of the surrounding medium. Neither of these can easily be included in theoretical studies. On the experimental side we have made extensive DATS measurements in various phases of both helium isotopes (room-temperature gas, lowtemperature gas, liquid and solid helium $[15,19,20])$ using antiprotons from the low-energy antiproton ring (LEAR) at CERN. The longest lifetime observed (about $4 \mu \mathrm{s}$ ) occurred in 10-K 1-bar helium gas. The difference between this and the liquid-helium value was surprisingly small as the $\bar{p} \mathrm{He}^{+}$atoms should be much less disturbed by collisions in lower than in higher-density media. We had, in effect, expected a marked increase in the average $\bar{p} \mathrm{He}^{+}$atom lifetime as these become more isolated, since then the $\bar{p}$ ought to be able to make more radiative transitions before reaching a short-lived state. This weak dependence of the average lifetime of $\bar{p}$ on the density of the helium means that the metastable atoms are extraordinarily insensitive to the presence of helium atoms.

On the other hand, our first LEAR experiments $[15,19]$ had already revealed that very small admixtures of hydrogen (even less than $100 \mathrm{ppm}$ ) destroy the metastability. This suggests that an entirely different quenching mechanism is at work when "foreign" atoms or molecules are present than when the $\bar{p} \mathrm{He}^{+}$atoms are surrounded exclusively by helium atoms. We have consequently made extensive studies at LEAR of DATS in room-temperature helium gas with admixtures of other noble gases $(\mathrm{He}, \mathrm{Ar}, \mathrm{Kr}, \mathrm{Xe})$ as well as molecular gases $\left(\mathrm{N}_{2}, \mathrm{O}_{2}, \mathrm{H}_{2}\right)$ and these form the subject matter of this paper. Section II below is devoted to the experimental setup, Sec. III to the analysis procedure, and Sec. IV to the experimental results. In Sec. V we present a detailed discussion of the data in terms of two model functions and in Sec. VI we draw some conclusions about the general features of the quenching effect on metastable $\bar{p} \mathrm{He}^{+}$of foreign atoms and molecules.

\section{EXPERIMENTAL SETUP}

\section{A. Particle detectors}

The method of measuring DATS in gaseous helium at LEAR has been already described in detail in a previous publication [19]. The current experiments have been performed using the same detector setup, the same beam, and the same $\bar{p}$ momentum $(200 \mathrm{MeV} / c)$ as those used in our liquid- and solid-helium experiments [20].

The experimental apparatus is shown in Fig. 1. The antiprotons left the beam line through a $100-\mu$ m-thick Be window and entered our target chamber (TC) after passing through a $1-\mathrm{mm}$-thick plastic scintillation counter $(B)$ of 10 $\mathrm{mm}$ diameter placed $18 \mathrm{~cm}$ downstream of the Be window. The $B$ counter was viewed by two photomultiplier tubes operated in coincidence. An additional ring-shaped counter $(A)$, which served as a veto counter as well as a monitor of the incoming beam halo, was placed about $1 \mathrm{~cm}$ upstream of $B$. The targets and the detectors for charged annihilation products (mainly pions) were located just downstream of the beam counters. The outgoing $\pi^{ \pm}$were detected by the same four sets of plastic scintillator telescopes used in previous experiments [19]. Each consisted of one inner $\left(T 1_{i}, i=\right.$ 


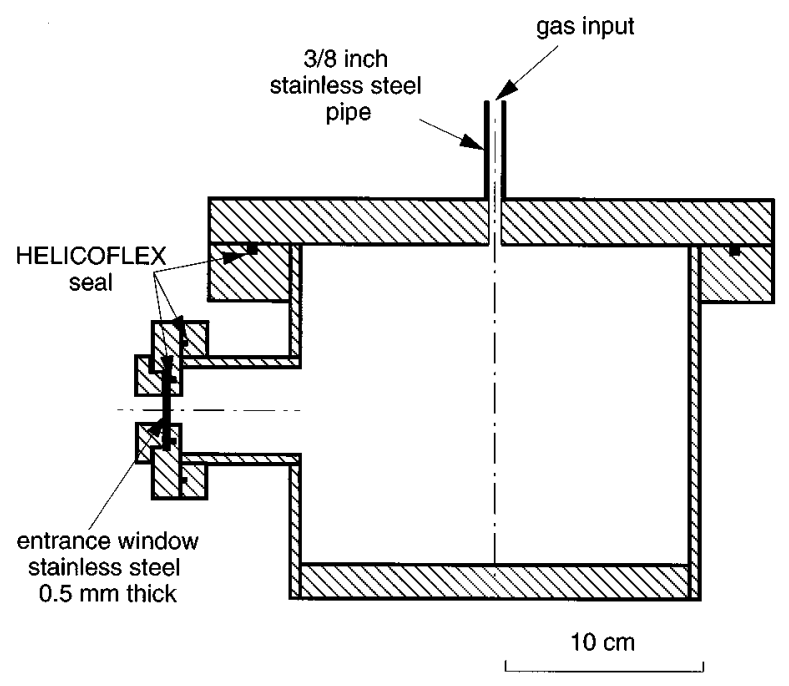

FIG. 2. Target-gas chamber. For details see the text.

$A, B, C, D$ in Fig. 1) and three outer $\left(T 2_{i j}, j=a, b, c\right)$ arrays, covering a solid angle of $0.8 \pi$, and was divided into 12 elements to reduce the probability of two or more pions hitting the same telescope counter. This was necessary to reduce the background, which is caused by $\pi^{+} \rightarrow \mu^{+} \rightarrow e^{+}$decay from pions stopped in the vicinity of the target chamber. These give a background with a lifetime of $2.2 \mu \mathrm{s}$, which lies within the time scale of interest. In order to reduce this background, it was found to be very effective to tag multipion hits in the telescope counters [19].

\section{B. High-pressure target-gas chamber}

Figure 2 shows the target-gas chamber. It consists of a vertical cylinder made of stainless steel (inner diameter 197 $\mathrm{mm}$, and length $190 \mathrm{~mm}$, and wall thickness $5 \mathrm{~mm}$ ) with a removable flange on top, through which it was connected to the gas mixing system. The entrance window for the $\bar{p}$ was mounted on a short cylinder of inner diameter $54.4 \mathrm{~mm}$, length $60 \mathrm{~mm}$, and wall thickness $3 \mathrm{~mm}$ perpendicular to the axis of the main cylinder. The window was made of a stainless-steel foil of a thickness of $0.5 \mathrm{~mm}$ held by a Helicoflex seal pressed against a flange. The inner diameter of the hole in the flange was $15 \mathrm{~mm}$. The use of Helicoflex seals permitted baking at temperatures up to $250{ }^{\circ} \mathrm{C}$.

The high pressure (up to $40 \mathrm{bar}$ ) at which the chamber was to be operated meant that the $\bar{p}$ entrance window had to be too small to accept the full antiproton beam, whose diameter was mainly determined by multiple scattering in the Be window and the upstream beam counter. Some particles therefore stopped inside the flange, making it impossible to give a reliable value for the absolute fraction of $\bar{p}$ trapped in metastable states. To stop the particles in 5-bar roomtemperature helium gas, an additional degrader of $0.5-\mathrm{mm}$ Kapton was necessary. Typical stopping distributions under these conditions were $15 \mathrm{~cm}$ (full width at half maximum) in range and $5 \mathrm{~cm}$ diameter perpendicular to the beam axis.

\section{Target-gas system}

The target-gas mixing system is shown in Fig. 3. It consists entirely of standard stainless-steel pipes of $3 / 8$ in. outer diameter connected by Swadgelok or Gyrolok joints. The valves were membrane-type valves suitable for baking at 250 ${ }^{\circ} \mathrm{C}$. The whole system including the target chamber was baked at that temperature for $8 \mathrm{~h}$ while evacuating it with two turbomolecular pumps (TMP1 and TMP2) before the experiment. The molecular sieve shown in the figure was included to trap impurities when cooled down by liquid nitrogen, but was found not to give a measurable improvement in the gas purity and was therefore not used in the experiments.

To mix the gas samples, we proceeded as follows. For high admixture concentrations, research grade admixture (impurity content between 3 and $20 \mathrm{ppm}$ ) gas was first introduced into the TC at partial pressure $p_{\mathrm{TC}}$. Research grade helium gas (impurity content $<1 \mathrm{ppm}$ ) was then added up to the total pressure $p_{t}$. The pressures were measured by a strain-gauge-type pressure sensor $(\mathrm{PG})$ with a resolution of 0.1 bar.

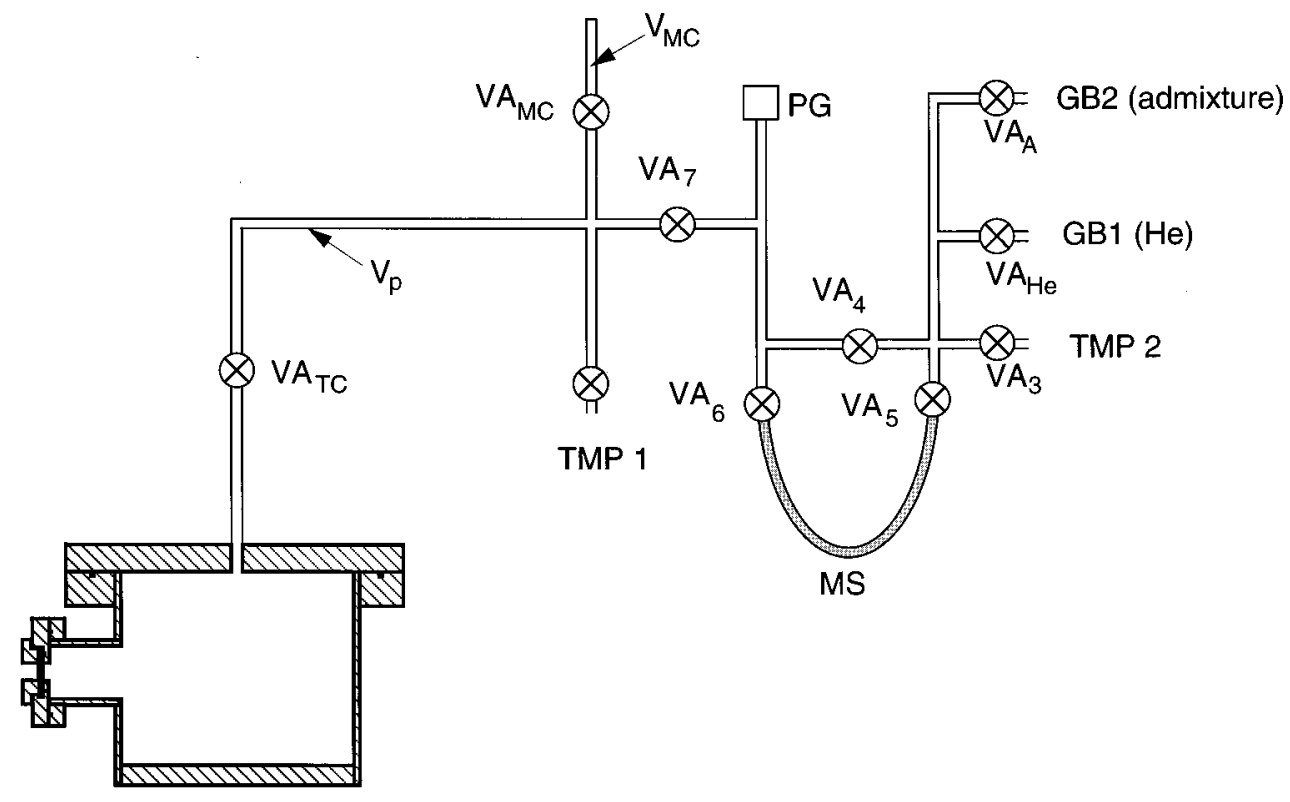

FIG. 3. System to mix the gas samples. Abbreviations are as follows: TC, target chamber; VA, valve; PG, pressure gauge; GB, gas bottle; TMP, turbomolecular pump; MS, molecular sieve. $V_{\mathrm{MC}}$ is the volume of the mixing chamber, $V_{p}$ the one of the pipes connecting MC and the target chamber TC. 


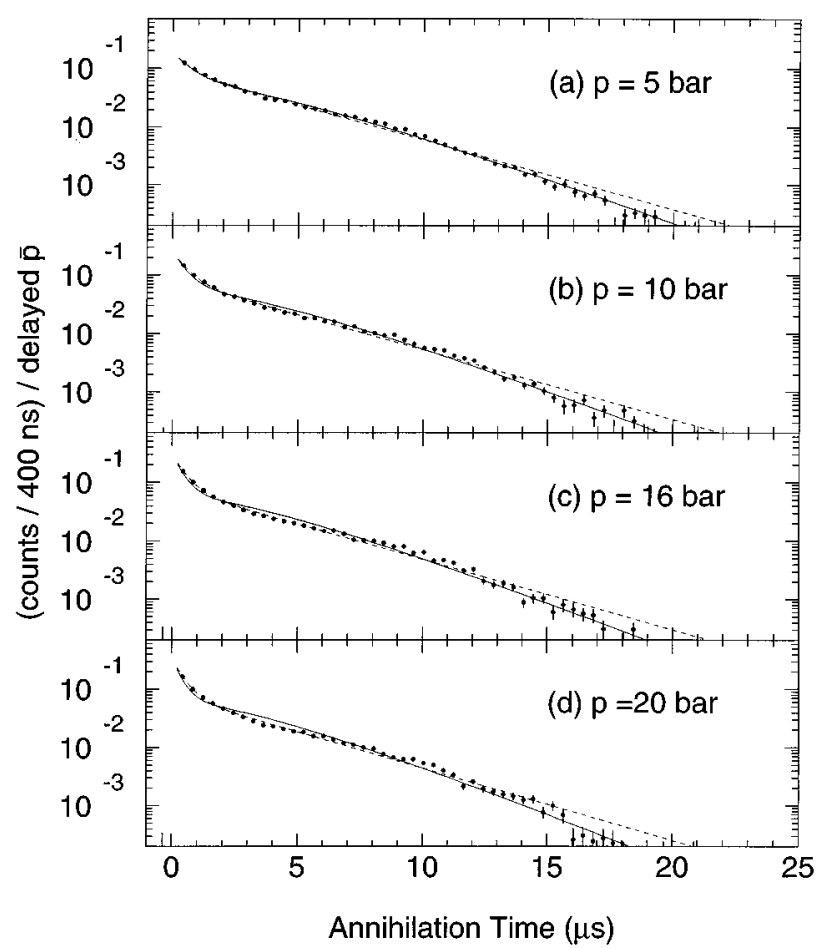

FIG. 4. DATS of pure helium at room temperature for several pressures. The data are shown as black dots, a solid line represents the fit of the three-level model, and the dashed line is the fit of a two-exponential model as described in the text.

In the case of low admixture concentrations, the foreign gas was first introduced into the mixing chamber (MC) at pressure $p_{\mathrm{MC}}$ and then the remaining pipe work, etc., was evacuated. Valve $\mathrm{VA}_{\mathrm{MC}}$ was opened to fill TC with admixture gas at partial pressure

$$
p_{a}=p_{\mathrm{MC}} V_{\mathrm{MC}} /\left(V_{\mathrm{TC}}+V_{p}\right) .
$$

The values for $V_{\mathrm{MC}}, V_{\mathrm{TC}}$, and $V_{p}$ were $10.3,5935$, and 120 $\mathrm{cm}^{3}$, respectively. The TC was then filled with helium gas to the final pressure $p_{t}$, yielding an admixture of concentration $c_{a}=p_{a} / p_{t}$. During data taking, the valve $\mathrm{VA}_{\mathrm{TC}}$ was closed and the rest of the system maintained at $10^{-5}$ mbar by pumping. In general, we first prepared a gas sample with the highest admixture concentration and took DATS over the appropriate range of pressures; $c_{a}$ was then successively reduced by adding pure helium gas.

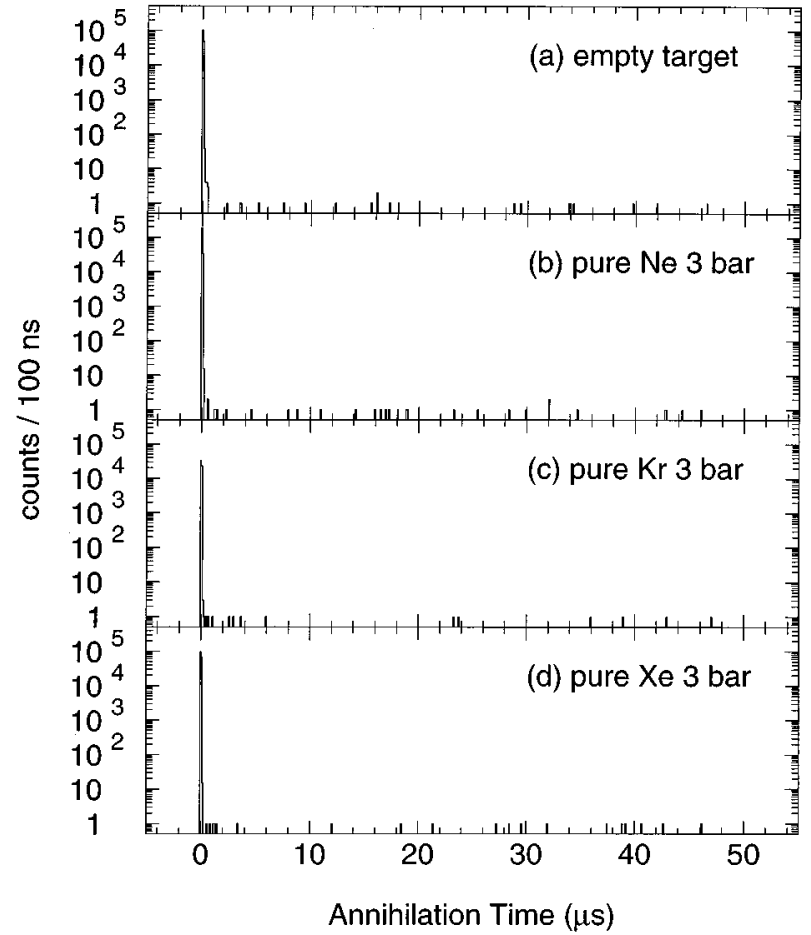

FIG. 5. M3 DATS of pure $\mathrm{Ne}, \mathrm{Kr}$, and $\mathrm{Xe}$ at 3 bar pressure and that for an empty target.

\section{DATA REDUCTION AND ANALYSIS}

The electronics, data taking, and data analysis procedure was described in great detail in two previous publications $[19,20]$. The time difference between an incoming $\bar{p}(B$ counter hit) and an outgoing annihilation product (a hit in at least one of the 12 telescope elements) was recorded separately for all 12 counters. This way we could select multipion hits in the off-line analysis to discriminate the background from the $\pi^{+} \rightarrow \mu^{+} \rightarrow e^{+}$decays. As described in [20], we used only events where at least two telescope counters registered a hit within a time window of $3.3 \mathrm{~ns}$ (multiplicity $\geqslant 2$ or $M 2$ events). To remove the remaining contamination of $\pi^{+} \rightarrow \mu^{+} \rightarrow e^{+}$decay in the $M 2$ spectra, we made a background run with empty target. The spectra presented in this paper were then obtained by subtracting the $M 2$ background spectrum from each data spectrum, normalized to the same total count. These spectra have also been normalized to the total delayed event count.

TABLE I. Summary of target conditions and fitted decay rates for pure helium gas. $p_{t}$ is the total pressure, $\lambda_{1}, \lambda_{3}, f, \lambda_{f}$, and $\lambda_{s}$ are defined in Eqs. (1)-(4).

\begin{tabular}{lccccc}
\hline \hline $\begin{array}{l}p_{t} \\
\text { (bar) }\end{array}$ & $\begin{array}{c}\lambda_{1} \\
(\mu \mathrm{s})^{-1}\end{array}$ & $\begin{array}{c}\lambda_{3} \\
(\mu \mathrm{s})^{-1}\end{array}$ & $f$ & $\begin{array}{c}\lambda_{f} \\
\left(\mu \mathrm{s}^{-1}\right)\end{array}$ & $\begin{array}{c}\lambda_{s} \\
\left(\mu \mathrm{s}^{-1}\right)\end{array}$ \\
\hline \multicolumn{5}{c}{ room temperature gas } \\
3.0 & $0.380 \pm 0.006$ & $0.98 \pm 0.03$ & $0.022 \pm 0.011$ & $1.72 \pm 0.66$ & $0.270 \pm 0.004$ \\
5.0 & $0.392 \pm 0.002$ & $1.44 \pm 0.02$ & $0.125 \pm 0.005$ & $1.83 \pm 0.09$ & $0.276 \pm 0.002$ \\
10.0 & $0.404 \pm 0.003$ & $2.01 \pm 0.03$ & $0.230 \pm 0.006$ & $1.92 \pm 0.07$ & $0.275 \pm 0.002$ \\
16.0 & $0.417 \pm 0.003$ & $2.39 \pm 0.05$ & $0.276 \pm 0.007$ & $2.13 \pm 0.08$ & $0.278 \pm 0.003$ \\
20.0 & $0.434 \pm 0.003$ & $2.65 \pm 0.06$ & $0.300 \pm 0.007$ & $2.29 \pm 0.08$ & $0.287 \pm 0.003$ \\
& & $T=470 \mathrm{~K}$ & & \\
5.0 & $0.423 \pm 0.007$ & $1.78 \pm 0.08$ & $0.171 \pm 0.017$ & $1.95 \pm 0.24$ & $0.296 \pm 0.007$ \\
\hline \hline
\end{tabular}


TABLE II. Summary of target conditions and fitted decay rates for noble-gas admixtures. $p_{a}$ is the partial pressure of the admixture gas, $p_{t}$ the total pressure, $f_{a}$ the admixture fraction, and $\rho_{a}$ the number of admixture atoms per $\mathrm{cm}^{2} . \lambda_{1}, \lambda_{3}, f, \lambda_{f}$, and $\lambda_{s}$ are defined in Eqs. (1)-(4).

\begin{tabular}{|c|c|c|c|c|c|c|c|c|}
\hline $\begin{array}{l}\mathrm{p}_{a} \\
\text { (mbar) }\end{array}$ & $\begin{array}{c}\mathrm{p}_{t} \\
\text { (bar) }\end{array}$ & $\begin{array}{l}\mathrm{f}_{a} \\
(\%)\end{array}$ & $\begin{array}{c}\rho_{a} \\
\left(10^{19} \mathrm{~cm}^{-3}\right)\end{array}$ & $\begin{array}{c}\lambda_{1} \\
(\mu s)^{-1}\end{array}$ & $\begin{array}{c}\lambda_{3} \\
(\mu s)^{-1}\end{array}$ & $f$ & $\begin{array}{c}\lambda_{f} \\
\left(\mu s^{-1}\right)\end{array}$ & $\begin{array}{c}\lambda_{s} \\
\left(\mu s^{-1}\right)\end{array}$ \\
\hline \multicolumn{9}{|c|}{ neon } \\
\hline 500 & 5.0 & 10 & 1.34 & $0.45 \pm 0.01$ & $1.53 \pm 0.10$ & $0.37 \pm 0.12$ & $0.80 \pm 0.20$ & $0.21 \pm 0.04$ \\
\hline 1000 & 10.0 & 10 & 2.69 & $0.54 \pm 0.01$ & $2.39 \pm 0.15$ & $0.32 \pm 0.04$ & $1.53 \pm 0.21$ & $0.31 \pm 0.02$ \\
\hline 1000 & 5.0 & 20 & 2.69 & $0.42 \pm 0.01$ & $1.52 \pm 0.08$ & $0.17 \pm 0.03$ & $1.45 \pm 0.32$ & $0.27 \pm 0.01$ \\
\hline 1500 & 15.0 & 10 & 4.03 & $0.60 \pm 0.01$ & $2.99 \pm 0.20$ & $0.53 \pm 0.05$ & $1.36 \pm 0.14$ & $0.27 \pm 0.03$ \\
\hline 2000 & 10.0 & 20 & 5.38 & $0.51 \pm 0.01$ & $2.32 \pm 0.13$ & $0.29 \pm 0.03$ & $1.63 \pm 0.19$ & $0.31 \pm 0.01$ \\
\hline 3000 & 15.0 & 20 & 8.07 & $0.59 \pm 0.01$ & $2.81 \pm 0.16$ & $0.34 \pm 0.03$ & $1.83 \pm 0.18$ & $0.35 \pm 0.01$ \\
\hline 7600 & 15.0 & 50.7 & 20.4 & $0.64 \pm 0.03$ & $3.42 \pm 0.45$ & $0.54 \pm 0.27$ & $1.35 \pm 0.69$ & $0.32 \pm 0.19$ \\
\hline \multicolumn{9}{|c|}{ argon } \\
\hline 17 & 15.3 & 0.11 & 0.045 & $0.39 \pm 0.01$ & $3.16 \pm 0.27$ & $0.32 \pm 0.02$ & $3.19 \pm 0.36$ & $0.25 \pm 0.01$ \\
\hline 300 & 3.0 & 10 & 0.81 & $0.43 \pm 0.01$ & $2.07 \pm 0.19$ & $0.18 \pm 0.02$ & $2.74 \pm 0.55$ & $0.27 \pm 0.01$ \\
\hline 600 & 3.0 & 20 & 1.61 & $0.49 \pm 0.01$ & $3.10 \pm 0.20$ & $0.27 \pm 0.02$ & $3.15 \pm 0.36$ & $0.32 \pm 0.01$ \\
\hline 1000 & 5.0 & 20 & 2.69 & $0.55 \pm 0.01$ & $3.25 \pm 0.20$ & $0.32 \pm 0.02$ & $2.65 \pm 0.27$ & $0.34 \pm 0.01$ \\
\hline 1500 & 15.0 & 10 & 4.03 & $0.71 \pm 0.02$ & $4.56 \pm 0.40$ & $0.39 \pm 0.03$ & $3.77 \pm 0.55$ & $0.41 \pm 0.03$ \\
\hline 2000 & 10.0 & 20 & 5.38 & $0.67 \pm 0.01$ & $4.04 \pm 0.26$ & $0.37 \pm 0.02$ & $3.15 \pm 0.35$ & $0.43 \pm 0.02$ \\
\hline 3000 & 15.0 & 20 & 8.07 & $0.89 \pm 0.02$ & $4.99 \pm 0.33$ & $0.39 \pm 0.02$ & $4.10 \pm 0.51$ & $0.58 \pm 0.02$ \\
\hline \multicolumn{9}{|c|}{$\operatorname{argon} T=470 \mathrm{~K}$} \\
\hline 250 & 2.5 & 10 & 0.43 & $0.57 \pm 0.02$ & $3.19 \pm 0.32$ & $0.48 \pm 0.12$ & $1.42 \pm 0.43$ & $0.27 \pm 0.09$ \\
\hline 500 & 5.0 & 10 & 0.85 & $0.82 \pm 0.02$ & $4.71 \pm 0.36$ & $0.36 \pm 0.03$ & $3.29 \pm 0.53$ & $0.50 \pm 0.02$ \\
\hline 1000 & 10.0 & 10 & 1.71 & $1.01 \pm 0.02$ & $5.15 \pm 0.37$ & $0.45 \pm 0.05$ & $2.80 \pm 0.44$ & $0.58 \pm 0.04$ \\
\hline 1000 & 5.0 & 20 & 1.71 & $1.01 \pm 0.03$ & $5.53 \pm 0.49$ & $0.40 \pm 0.03$ & $4.22 \pm 0.80$ & $0.61 \pm 0.04$ \\
\hline 2000 & 10.0 & 20 & 3.41 & $1.66 \pm 0.06$ & $7.37 \pm 0.63$ & $0.47 \pm 0.07$ & $5.41 \pm 1.63$ & $1.35 \pm 0.14$ \\
\hline 3000 & 15.0 & 20 & 5.12 & $2.38 \pm 0.05$ & $8.79 \pm 0.63$ & $0.72 \pm 0.05$ & $3.33 \pm 0.31$ & $0.95 \pm 0.11$ \\
\hline \multicolumn{9}{|c|}{ krypton } \\
\hline 84 & 3.0 & 2.8 & 0.23 & $0.40 \pm 0.02$ & $1.15 \pm 0.12$ & $0.17 \pm 0.13$ & $0.91 \pm 0.46$ & $0.23 \pm 0.04$ \\
\hline 132 & 2.5 & 5.3 & 0.35 & $0.45 \pm 0.01$ & $1.73 \pm 0.13$ & $0.29 \pm 0.05$ & $1.17 \pm 0.17$ & $0.25 \pm 0.02$ \\
\hline 263 & 5.0 & 5.3 & 0.71 & $0.59 \pm 0.01$ & $2.98 \pm 0.20$ & $0.41 \pm 0.03$ & $1.82 \pm 0.19$ & $0.31 \pm 0.02$ \\
\hline 300 & 3.0 & 10 & 0.81 & $0.94 \pm 0.03$ & $4.85 \pm 0.48$ & $0.52 \pm 0.04$ & $2.56 \pm 0.34$ & $0.44 \pm 0.05$ \\
\hline 420 & 15.0 & 2.8 & 1.13 & $0.68 \pm 0.03$ & $3.35 \pm 0.37$ & $0.47 \pm 0.07$ & $2.01 \pm 0.39$ & $0.35 \pm 0.08$ \\
\hline 500 & 5.0 & 10 & 1.34 & $1.08 \pm 0.04$ & $4.92 \pm 0.45$ & $0.51 \pm 0.05$ & $3.01 \pm 0.36$ & $0.59 \pm 0.06$ \\
\hline 526 & 10.0 & 5.3 & 1.42 & $0.78 \pm 0.02$ & $4.29 \pm 0.26$ & $0.49 \pm 0.02$ & $3.00 \pm 0.25$ & $0.43 \pm 0.03$ \\
\hline 789 & 15.0 & 5.3 & 2.12 & $1.02 \pm 0.02$ & $5.19 \pm 0.26$ & $0.57 \pm 0.02$ & $3.30 \pm 0.21$ & $0.52 \pm 0.03$ \\
\hline 1000 & 19.0 & 5.3 & 2.69 & $1.31 \pm 0.04$ & $6.28 \pm 0.45$ & $0.61 \pm 0.02$ & $4.50 \pm 0.38$ & $0.58 \pm 0.05$ \\
\hline 1000 & 10.0 & 10 & 2.69 & $1.40 \pm 0.06$ & $6.59 \pm 0.56$ & $0.67 \pm 0.03$ & $5.24 \pm 0.53$ & $0.77 \pm 0.08$ \\
\hline 1500 & 15.0 & 10 & 4.03 & $2.56 \pm 0.01$ & $9.03 \pm 1.00$ & $0.74 \pm 0.02$ & $7.63 \pm 0.68$ & $0.84 \pm 0.07$ \\
\hline \multicolumn{9}{|c|}{ xenon } \\
\hline 1.25 & 5.0 & 0.025 & $3.36 \times 10^{-3}$ & $0.85 \pm 0.04$ & $6.19 \pm 0.50$ & $0.80 \pm 0.03$ & $12.15 \pm 1.30$ & $0.65 \pm 0.05$ \\
\hline 1.25 & 2.5 & 0.05 & $3.36 \times 10^{-3}$ & $1.44 \pm 0.09$ & $7.34 \pm 0.93$ & $0.70 \pm 0.04$ & $7.34 \pm 1.25$ & $0.59 \pm 0.13$ \\
\hline 5.0 & 10.0 & 0.05 & $1.34 \times 10^{-2}$ & $1.06 \pm 0.06$ & $5.19 \pm 0.65$ & $0.40 \pm 0.11$ & $4.09 \pm 1.21$ & $1.06 \pm 0.16$ \\
\hline
\end{tabular}

\section{EXPERIMENTAL RESULTS}

Figure 4 shows DATS obtained in pure helium gas at room temperature for several pressures. Together with the data (black dots) the results of a fit of two model functions described in Sec. V are shown as solid and dashed lines, respectively. We chose a binning of $100 \mathrm{~ns}$ for all displayed spectra, though our intrinsic instrumental time resolution is 3 ns. The spectra exhibit a fast component and, as observed previously, a growth-type structure, which results in a downward-bent shape in the semilogarithmic plot at times $t \geqslant 10 \mu \mathrm{s}$. Previously recorded spectra of low-temperature helium gas [20] at temperatures below $30 \mathrm{~K}$ did not show such a fast component. However, as will be discussed later, we cannot exclude room-temperature impurities as the origin of this feature.

The multiplicity $\geqslant 3(M 3)$ DATS in pure $\mathrm{Ne}, \mathrm{Kr}$, and $\mathrm{Xe}$ at 3 bar are shown in Fig. 5 together with that of an empty target chamber run. They show only a prompt peak and randomly occurring delayed counts. The upper limit of about 5 $\times 10^{-4}$ for the delayed fraction is consistent with the value we obtain for the empty target run. Tables I-III list the conditions for pure helium as well as for all gas mixtures measured. 
TABLE III. Summary of target conditions and fitted decay rates for molecular mixture gases. $p_{a}$ is the partial pressure of the admixture gas, $p_{t}$ the total pressure, $f_{a}$ the admixture fraction, and $\rho_{a}$ the number of admixture atoms per $\mathrm{cm}^{2} . \lambda_{1}, \lambda_{3}, f, \lambda_{f}$, and $\lambda_{s}$ are defined in Eqs. (1)-(4).

\begin{tabular}{|c|c|c|c|c|c|c|c|c|}
\hline $\begin{array}{l}p_{a} \\
\text { (mbar) }\end{array}$ & $\begin{array}{c}p_{t} \\
\text { (bar) }\end{array}$ & $\begin{array}{c}f_{a} \\
(\mathrm{ppm})\end{array}$ & $\begin{array}{c}\rho_{a} \\
\left(10^{17} \mathrm{~cm}^{-3}\right)\end{array}$ & $\begin{array}{c}\lambda_{1} \\
(\mu s)^{-1}\end{array}$ & $\begin{array}{c}\lambda_{3} \\
(\mu \mathrm{s})^{-1}\end{array}$ & $f$ & $\begin{array}{c}\lambda_{f} \\
\left(\mu \mathrm{s}^{-1}\right)\end{array}$ & $\begin{array}{c}\lambda_{s} \\
\left(\mu \mathrm{s}^{-1}\right)\end{array}$ \\
\hline \multicolumn{9}{|c|}{ hydrogen } \\
\hline 0.63 & 2.5 & 250 & 0.168 & $3.01 \pm 0.07$ & $10.6 \pm 0.6$ & $0.77 \pm 0.03$ & $5.47 \pm 0.43$ & $1.15 \pm 0.09$ \\
\hline 2.50 & 2.5 & 1000 & 0.672 & $6.33 \pm 0.23$ & $20.0 \pm 4.8$ & $0.82 \pm 0.04$ & $10.7 \pm 1.8$ & $2.72 \pm 0.41$ \\
\hline 10.0 & 10.0 & 1000 & 2.69 & $13.3 \pm 1.0$ & $13.0 \pm 1.3$ & $1.04 \pm 0.01$ & $6.78 \pm 0.01$ & \\
\hline \multicolumn{9}{|c|}{ oxygen } \\
\hline 0.06 & 2.5 & 25 & 0.0168 & $0.77 \pm 0.01$ & $2.14 \pm 0.09$ & $0.40 \pm 0.10$ & $0.82 \pm 0.11$ & $0.46 \pm 0.04$ \\
\hline 0.30 & 12.3 & 25 & 0.0813 & $2.54 \pm 0.03$ & $8.61 \pm 0.86$ & $0.63 \pm 0.17$ & $2.36 \pm 0.26$ & $1.23 \pm 0.18$ \\
\hline 0.30 & 2.5 & 121 & 0.0813 & $2.55 \pm 0.04$ & $8.49 \pm 1.07$ & $0.35 \pm 0.09$ & $2.69 \pm 0.38$ & $1.53 \pm 0.07$ \\
\hline 1.25 & 10.3 & 121 & 0.336 & $8.29 \pm 0.18$ & $21.6 \pm 5.6$ & $1.00 \pm 0.02$ & $6.06 \pm 0.12$ & \\
\hline 1.25 & 2.5 & 500 & 0.336 & $9.77 \pm 0.14$ & $10.2 \pm 0.3$ & $1.00 \pm 0.03$ & $5.98 \pm 0.12$ & \\
\hline \multicolumn{9}{|c|}{ nitrogen } \\
\hline 2.42 & 2.5 & 970 & 0.651 & $0.50 \pm 0.01$ & $3.54 \pm 0.17$ & $0.41 \pm 0.01$ & $2.68 \pm 0.20$ & $0.29 \pm 0.01$ \\
\hline 9.98 & 10.3 & 970 & 2.68 & $0.75 \pm 0.02$ & $4.85 \pm 0.26$ & $0.58 \pm 0.02$ & $3.58 \pm 0.25$ & $0.37 \pm 0.02$ \\
\hline
\end{tabular}

A selection of admixture DATS is shown in Figs. 6-13. They can generally be described rather well by a simple superposition of two exponential curves (dashed lines). The higher the admixture concentration, the steeper and more pronounced the fast component becomes, while at the same time the slow component lifetime becomes shorter. A given admixture concentration shortens the average lifetime significantly more at high temperature than at room temperature (see Figs. 7 and 8 for Ar). It also appears that the sensitivity of the average lifetime to admixture concentration varies considerably from gas to gas: even at $20 \%$ concentration, $\mathrm{Ne}$, $\mathrm{Ar}$, and $\mathrm{Kr}$ change the average lifetime only slightly, while

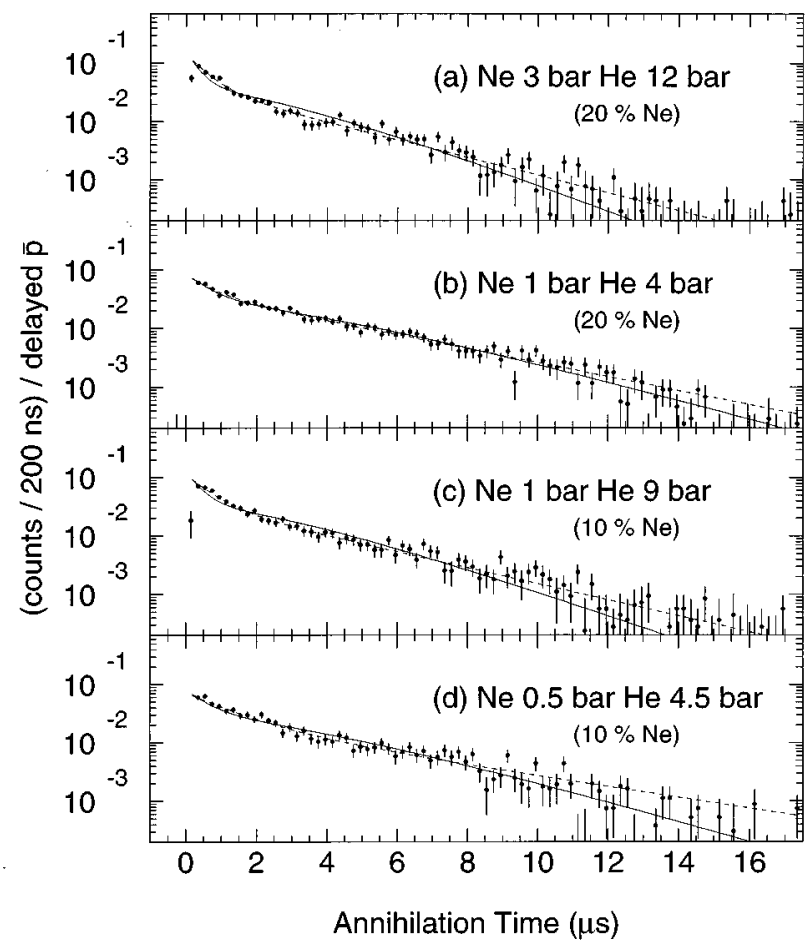

FIG. 6. DATS of helium with an admixture of Ne. the shape of the DATS is significantly affected by Xe and by molecular impurities at concentrations less than $0.1 \%$. Among the molecular impurities, there is also some variation: $\mathrm{N}_{2}$ in concentrations of $1000 \mathrm{ppm}$ affects the average lifetime of $\bar{p}$ much less than $\mathrm{H}_{2}$ or $\mathrm{O}_{2}$ at the 100-ppm level.

\section{DISCUSSION}

\section{A. Descriptive models of the time spectra}

In order to get quantitative results on the change of the time spectra due to admixtures it is necessary to describe the observed spectra by some model functions. In this paper we have used two of these.

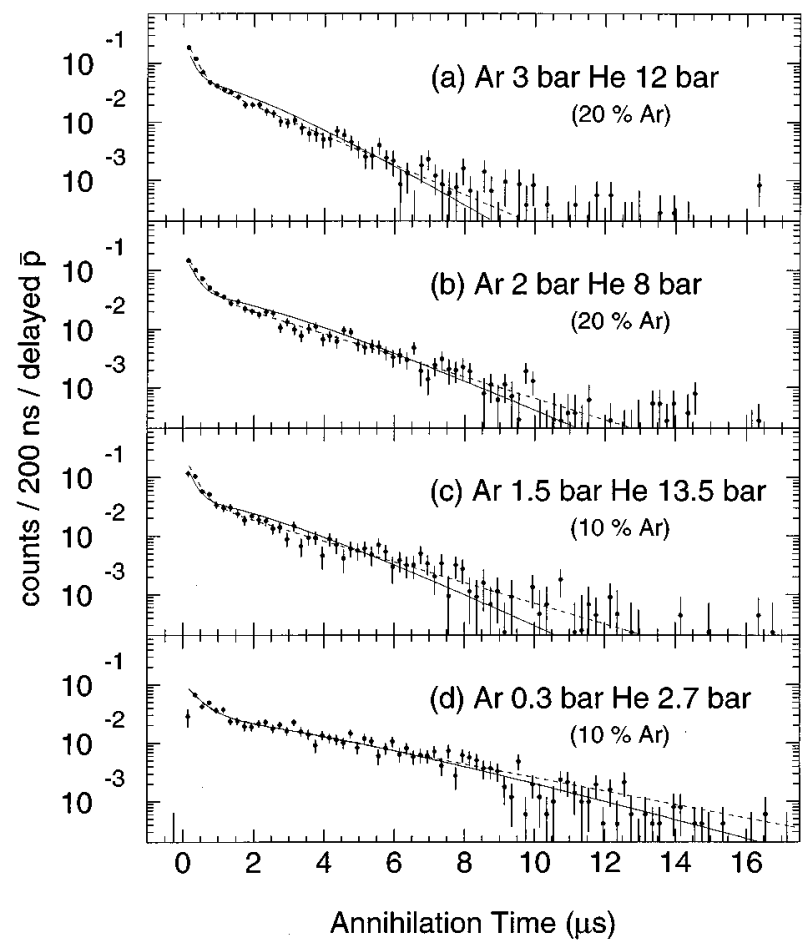

FIG. 7. DATS of helium with an admixture of Ar. 


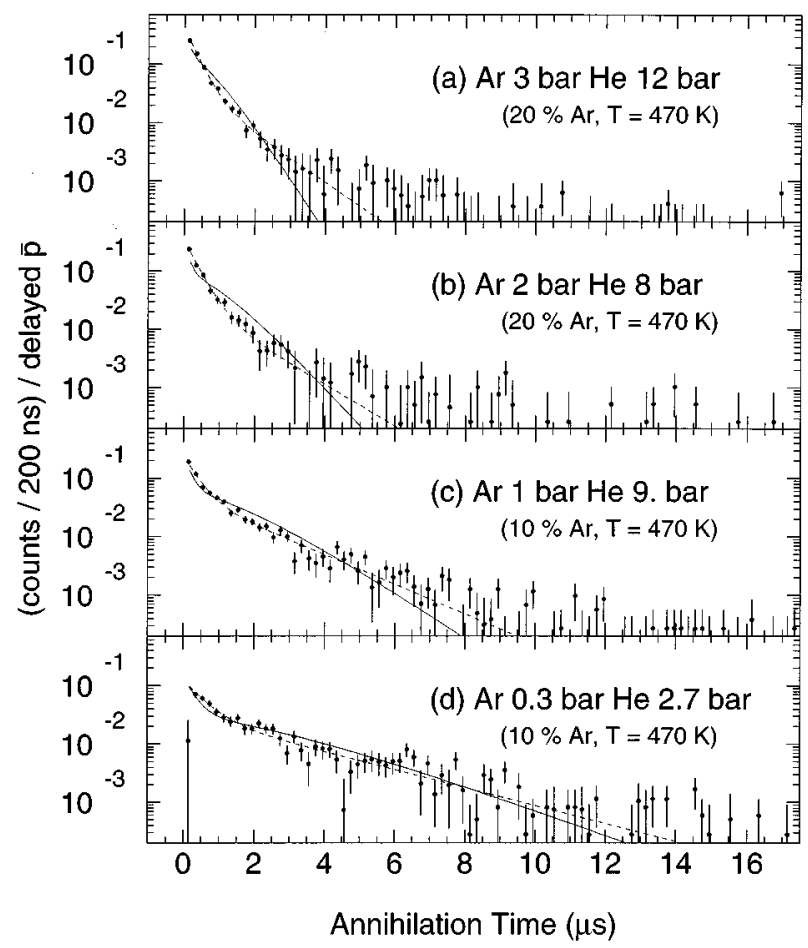

FIG. 8. DATS of helium with an admixture of Ar at $T=470$.

\section{Chain-decay model}

In a previous publication dealing with DATS for pure helium [20] we introduced a chain decay model in which the antiprotons pass through a sequence of three atomic states and annihilate rapidly with the nucleus from the lowest one. This model very successfully reproduced DATS in a range of

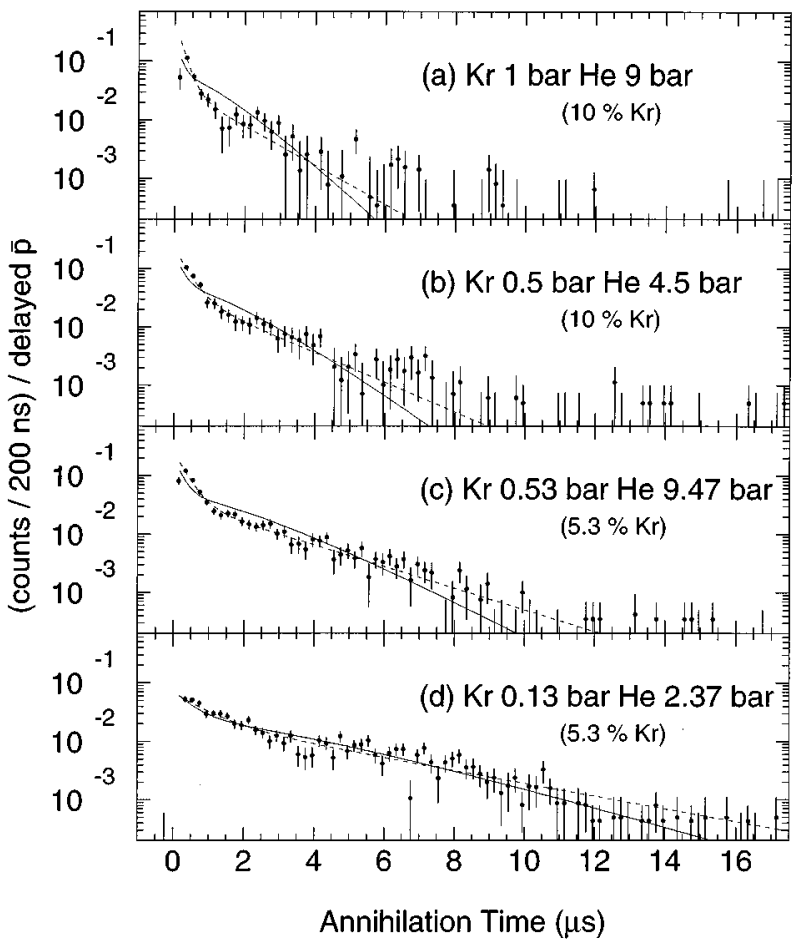

FIG. 9. DATS of helium with an admixture of $\mathrm{Kr}$.

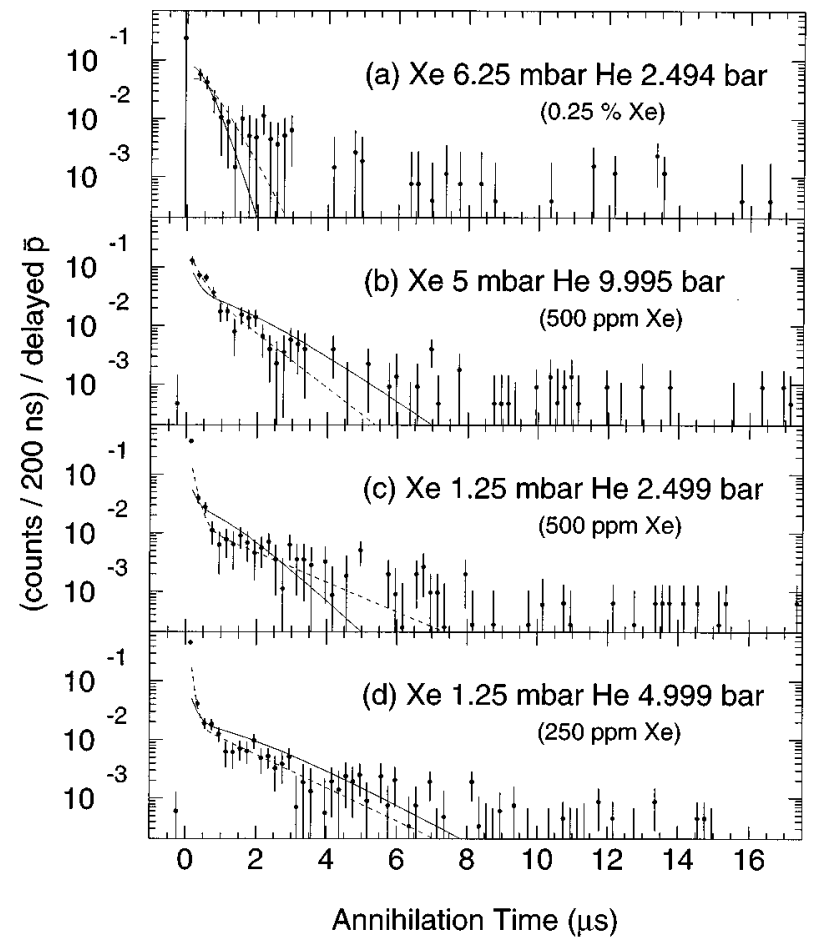

FIG. 10. DATS of helium with an admixture of Xe.

conditions from low-temperature gas to liquid and solid helium. Denoting $N_{1}, N_{2}, N_{3}$ the populations and $\lambda_{1}, \lambda_{2}, \lambda_{3}$ the decay rates of the three levels (level 1 is the highest in the chain), we can write down the rate equations for the timedependent populations

$$
\begin{gathered}
\frac{d N_{1}}{d t}=-\lambda_{1} N_{1}, \\
\frac{d N_{2}}{d t}=\lambda_{1} N_{1}-\lambda_{2} N_{2}, \\
\frac{d N_{3}}{d t}=\lambda_{2} N_{2}-\lambda_{3} N_{3} .
\end{gathered}
$$

Making the same simplifying assumptions as before $\left[N_{1}(0)=N_{2}(0)=N_{3}(0), \lambda_{2}=\lambda_{3}\right]$, we obtain a solution for

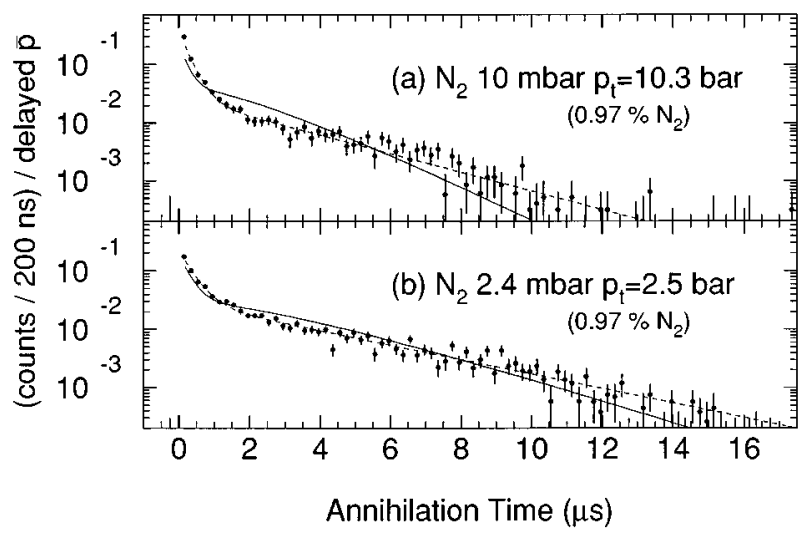

FIG. 11. DATS of helium with an admixture of nitrogen. 


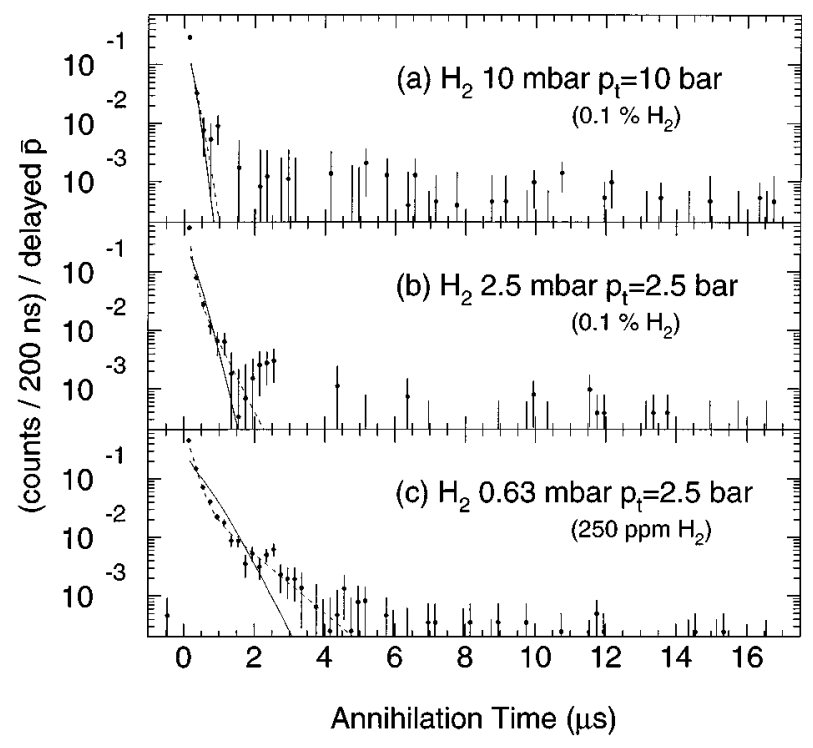

FIG. 12. DATS of helium with an admixture of hydrogen.

$N_{3}(t)$. The assumption that annihilation takes place only from the lowest level 3 means that we fit $\lambda_{3} N_{3}$ to our data. The results of $\log$ likelihood fits of this function to our DATS are shown as a solid line in Figs. 7-13.

While this model works well with noble-gas admixtures at low concentrations, its performance deteriorates (a) at high noble-gas concentrations and especially (b) if the impurities are molecular gases, it being unable to reproduce the steep fast component present in this case. Tables I-III give the results for the decay rates $\lambda_{1}$ and $\lambda_{3}$.

\section{Two-exponential model}

In view of the low statistical significance of some of the DATS, especially those at high admixture concentrations, we decided to try a very simple model, in which the spectra consisted only of a sum of independent exponential functions

$$
n(t)=A\left[f \lambda_{f} \exp \left(-\lambda_{f} t\right)+(1-f) \lambda_{s} \exp \left(-\lambda_{s} t\right)\right],
$$

where $A$ is an overall normalization, $\lambda_{f}$ and $\lambda_{s}$ are the fast and slow decay rates, and $f$ is the fraction of the fast component. The results of the fits of this function are shown as a dashed line in Figs. 7-13 and give a better description of the DATS at higher admixture concentrations than does the chain-decay model.

\section{B. Pure helium gas}

The fast component visible in pure helium, roomtemperature DATS was not observed in previous experiments at temperatures below $30 \mathrm{~K}$ [20], although it did appear above that temperature. Furthermore, at $50 \mathrm{~K}$ the delayed component consisted only of a single exponential with a lifetime of about 500 ns. This was attributed to the presence of an unknown impurity that entered the vapour phase above $30 \mathrm{~K}$. Even though we carefully baked the whole gas system and the target chamber in the present experiments, we cannot eliminate residual impurities at the 10ppm level. Our experiments with controlled concentrations of helium with hydrogen had already shown that these gases can have a marked effect on the delayed component even in concentrations of a few tens of ppm.

On the other hand, the fit results of the three-level model for the slow component $\left(\lambda_{1}\right)$ are in good agreement with the results for low-temperature gas (a typical value being $\lambda_{1}$ $=0.395 \pm 0.005 \mu \mathrm{s}^{-1}$ at $T=10 \mathrm{~K}$ [20]). Also the value of $\lambda_{1}$ does not change significantly between room temperature and $470 \mathrm{~K}$ (see Table I). We therefore mainly use the values of the slow component for the further discussion presented below.

\section{Noble-gas admixtures}

Figure 14 shows the fit results for $\lambda_{f}, \lambda_{s}$, and the fast component fraction $f$ versus the admixture density. The Xe data are included twice, for comparison with noble-gas values (left column) and molecular-gas values (right column). The relation is linear with density, the slope of straight line fits to the data increasing systematically from $\mathrm{Ne}$ to Xe. It is clear that the Xe slopes are very much higher than those for other noble gases and similar to those for nitrogen. In all cases the fitted lines extrapolate reasonably well to the values obtained for pure helium gas.

The fast component fraction $f$, shown in the lower part of Fig. 14, increases systematically with increasing admixture density, except for Xe. However, the large errors and sparse data points make it difficult to draw firm conclusions from this.

Since the fitted decay rates exhibit a fairly linear relation with the number density of the admixture, it is justified to apply a simple quenching model where the metastability is affected by binary collisions with the admixture atoms or molecules. This picture is justified if we assume that the quenching takes place after the formation of the metastable states. In addition, we assume that the metastable $\bar{p} \mathrm{He}^{+}$atoms are thermalized after their formation in a time scale short compared with the annihilation time scale. The decay rate dependence of $\lambda$ on the admixture density $n$ is

$$
\lambda=\lambda_{0}+\sigma_{q} v n
$$

where $\lambda_{0}$ is the intrinsic decay rate of the metastable state, $\sigma_{q}$ the quenching cross section and $v$ the velocity of the admixture atom or molecule relative to the $\bar{p} \mathrm{He}^{+}$atomcule. With our assumption that the $p \mathrm{He}^{+}$velocity is thermal, we get

$$
v=\sqrt{\frac{3 k T}{\mu}},
$$

where

$$
\mu=\frac{m_{a} M}{m_{a}+M}
$$

is the reduced mass of the system ( $M$ is the mass of $\bar{p} \mathrm{He}^{+}$system, and $m_{a}$ is the mass of admixture atom or molecule). Table IV gives the results of the fits shown as straight lines in Figs. 14 and 15.

It is remarkable that the values for $\sigma_{q}$ resulting from the two-level model are systematically smaller (by a factor 2-3) than that obtained from the three-level model. The general 
tendency with respect to the influence of different admixtures as described above is, however, similar in both models.

Figure 15 compares the density dependence of the fitted decay rates and fast component fraction of room temperature (RT) helium-plus-argon mixtures and the same mixture at a temperature $T=470 \mathrm{~K}$ ("hot" Ar). As can be seen in the left column, in both cases the decay rates depend linearly on the density. The slope for hot Ar is much steeper than for RT Ar mixtures. While the fraction for RT mixtures is fairly constant, that at higher temperatures increases steadily with admixture density.

The right column of Fig. 15 shows the data plotted against $\rho_{\text {admix }} \sqrt{T}$. If the difference of RT and hot mixtures were due only to the higher thermal velocity $(\propto \sqrt{T})$, the two data sets should lie on the same curve in this display. The fact that the slopes are still very different shows that the quenching cross section changes with temperature. If we assume an Arrhenius-type temperature dependence, i.e., $\sigma_{q}$ $\propto \exp \left(-E_{a} / k T\right)$, we can extract a value for the "activation energy" $E_{a}$ of $0.11 \pm 0.01 \mathrm{eV}$ in the three-level model and $0.073 \pm 0.008 \mathrm{eV}$ in the two-exponential model.

\section{Admixtures of molecular gases}

The right column of Fig. 14 shows the density dependence of the fitted decay rates for admixtures of $\mathrm{O}_{2}, \mathrm{H}_{2}$, and $\mathrm{N}_{2}$. The scale of the density axes is about three orders of magnitude smaller than the one for the noble gases (left column), indicating that the quenching effects are much stronger here. The data can (at least for the two-exponential model) again be well fitted by a straight line. The results of these fits are shown in Table IV. While the value of $\sigma_{q}$ for $\mathrm{N}_{2}$ is similar to that of $\mathrm{Xe}$, the values for $\mathrm{H}_{2}$ and $\mathrm{O}_{2}$ are one and two orders of magnitudes higher, respectively. For these

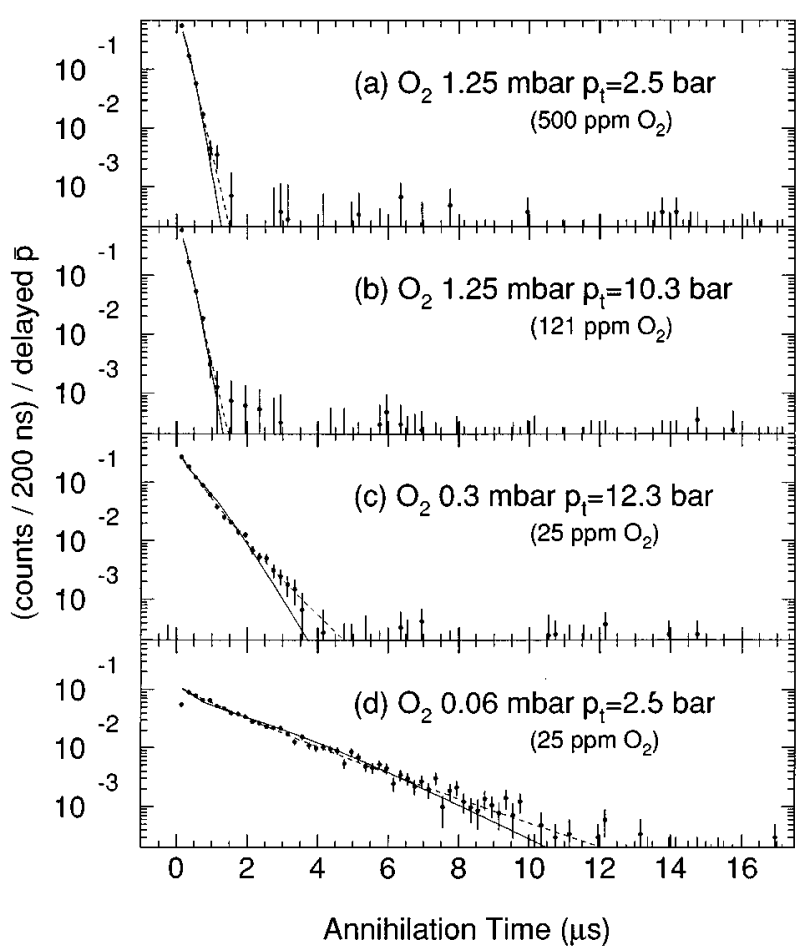

FIG. 13. DATS of helium with an admixture of oxygen.

molecular admixtures, the fast component fraction rises rapidly to the value unity with admixture density (for $\mathrm{O}_{2}$ even at $120 \mathrm{ppm}$ concentration). Effectively, at higher admixture concentrations the DATS is a single exponential.

\section{CONCLUSION}

In summary, we obtained DATS of helium with admixtures of other noble gases as well as molecular gases. Both
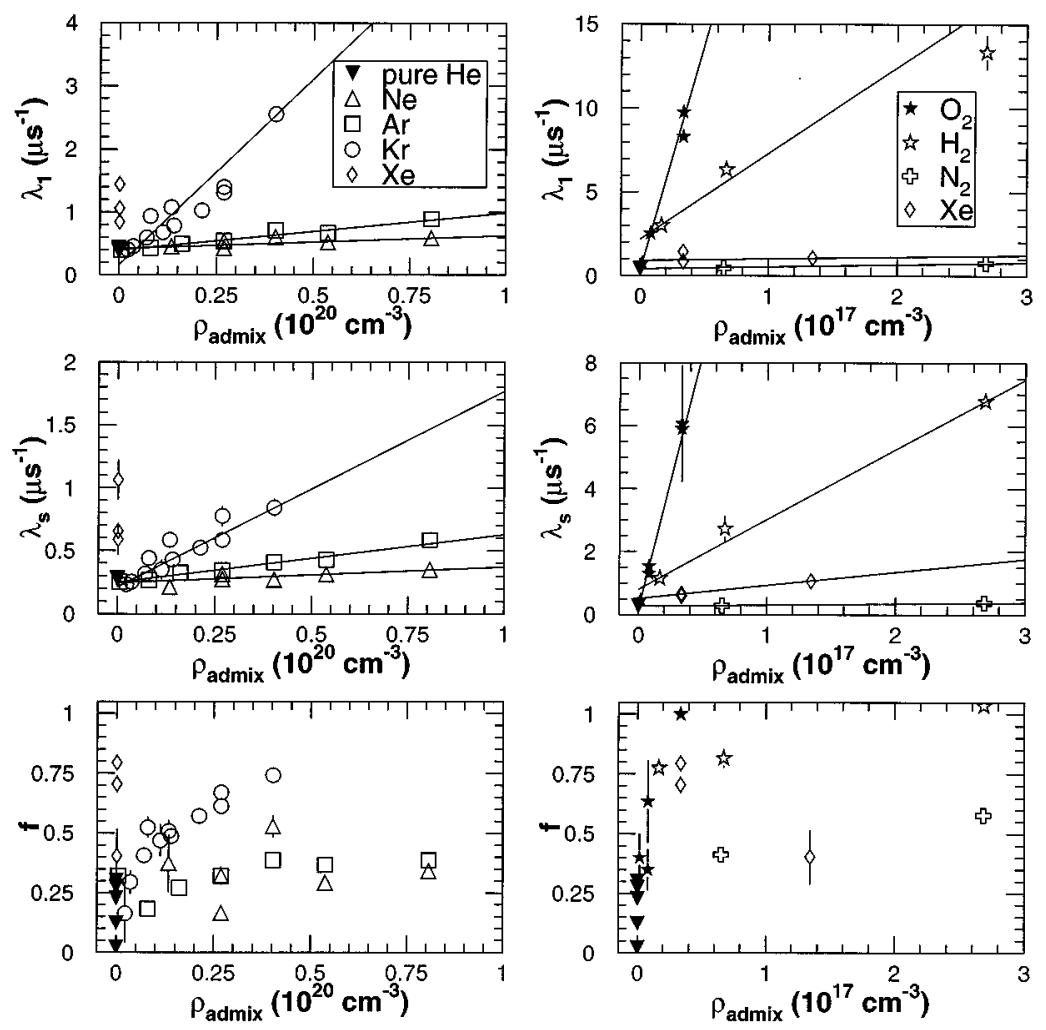

FIG. 14. Density dependence of $\lambda_{1}, \lambda_{s}$, and $f$ for noble-gas admixtures (left column) and molecular admixtures (right column). 
TABLE IV. Results of fits of the quenching model. The asterisk denotes $T=470 \mathrm{~K}$.

\begin{tabular}{cccccc}
\hline \hline & & \multicolumn{2}{c}{ Three-level model } & \multicolumn{2}{c}{ Two-level model } \\
Type & $v_{\text {th }}\left(10^{5} \mathrm{~cm} / \mathrm{s}^{-1}\right)$ & $\lambda_{0}\left(\mu \mathrm{s}^{-1}\right)$ & $\sigma_{q}\left(\mathrm{~cm}^{2}\right)$ & $\lambda_{0}\left(\mu \mathrm{s}^{-1}\right)$ & $\sigma_{q}\left(\mathrm{~cm}^{2}\right)$ \\
\hline $\mathrm{Ne}$ & $1.37 \pm 0.03$ & $0.421 \pm 0.010$ & $(1.5 \pm 0.2) \times 10^{-20}$ & $0.25 \pm 0.02$ & $(9 \pm 2) \times 10^{-21}$ \\
$\mathrm{Ar}$ & $1.30 \pm 0.02$ & $0.390 \pm 0.007$ & $(4.6 \pm 0.3) \times 10^{-20}$ & $0.249 \pm 0.008$ & $(2.9 \pm 0.2) \times 10^{-20}$ \\
$\mathrm{Ar}{ }^{*}$ & $1.63 \pm 0.10$ & $0.441 \pm 0.016$ & $(2.2 \pm 0.2) \times 10^{-19}$ & $0.37 \pm 0.03$ & $(8 \pm 2) \times 10^{-20}$ \\
$\mathrm{Kr}$ & $1.26 \pm 0.02$ & $0.164 \pm 0.009$ & $(4.7 \pm 0.1) \times 10^{-19}$ & $0.21 \pm 0.02$ & $(1.2 \pm 0.1) \times 10^{-19}$ \\
$\mathrm{Xe}$ & $1.25 \pm 0.02$ & & & $0.51 \pm 0.08$ & $(3 \pm 1) \times 10^{-18}$ \\
$\mathrm{~N}_{2}$ & $1.33 \pm 0.02$ & $0.414 \pm 0.015$ & $(9 \pm 1) \times 10^{-18}$ & $0.26 \pm 0.02$ & $(3 \pm 1) \times 10^{-18}$ \\
$\mathrm{H}_{2}$ & $2.28 \pm 0.04$ & $2.201 \pm 0.094$ & $(2.2 \pm 0.2) \times 10^{-16}$ & $0.79 \pm 0.10$ & $(9.7 \pm 0.3) \times 10^{-17}$ \\
$\mathrm{O}_{2}$ & $1.31 \pm 0.02$ & $0.323 \pm 0.015$ & $(2.05 \pm 0.06) \times 10^{-15}$ & $0.18 \pm 0.04$ & $(1.2 \pm 0.1) \times 10^{-15}$ \\
\hline \hline
\end{tabular}

admixtures shortened the average lifetime of DATS and introduced a fast-decaying component into the spectra as compared to pure helium at low temperatures. Pure helium measured at room temperature in the same target chamber, however, also showed a fast component of varying steepness. It cannot be excluded that this fast component is at least partially caused by the presence of impurities.

The most striking result was the difference in the effects of noble-gas and molecular-gas admixtures. The introduction of $\mathrm{Ne}, \mathrm{Ar}$, and $\mathrm{Kr}$ in concentrations of $10 \%$ changed the DATS only slightly. The fitted decay rates of either a threelevel model or a simple two-exponential model show a linear dependence on the admixture density, corresponding to a quenching cross section of the order of $10^{-20} \mathrm{~cm}^{2}$ if the $\bar{p} \mathrm{He}^{+}$atom are assumed to have thermal velocity.

Both $\mathrm{Xe}$ and $\mathrm{N}_{2}$ quenched the metastable states much more strongly, with cross sections of about $10^{-18} \mathrm{~cm}^{2}$, while $\mathrm{O}_{2}$ and $\mathrm{H}_{2}$ quenched them very violently in concen- trations of only $100 \mathrm{ppm}$. The latter figure corresponds to a quenching cross section of about $10^{-16} \mathrm{~cm}^{2}$, which is of the order of a typical "geometrical" atomic collision cross section. A single collision of the $\bar{p} \mathrm{He}^{+}$atom with an $\mathrm{O}_{2}$ or a $\mathrm{H}_{2}$ molecule is thus able to destroy the metastability completely. The reason for this may be that due to their many internal degrees of freedom, such molecules can, during a collision, easily absorb the exact amount of energy and angular momentum required to move the $\bar{p}$ from a metastable to a short-lived state. An alternative explanation might be the occurrence of some kind of chemical reaction. This could explain why the chemically less active $\mathrm{N}_{2}$ has a weaker effect on the atom.

The behavior of the quenching rate of argon between room temperature and $470 \mathrm{~K}$ seems to be attributable to a change in the cross section itself with temperature. Assuming an Arrhenius-type temperature dependence, we can extract an activation energy of about $0.1 \mathrm{eV}$ from these data.
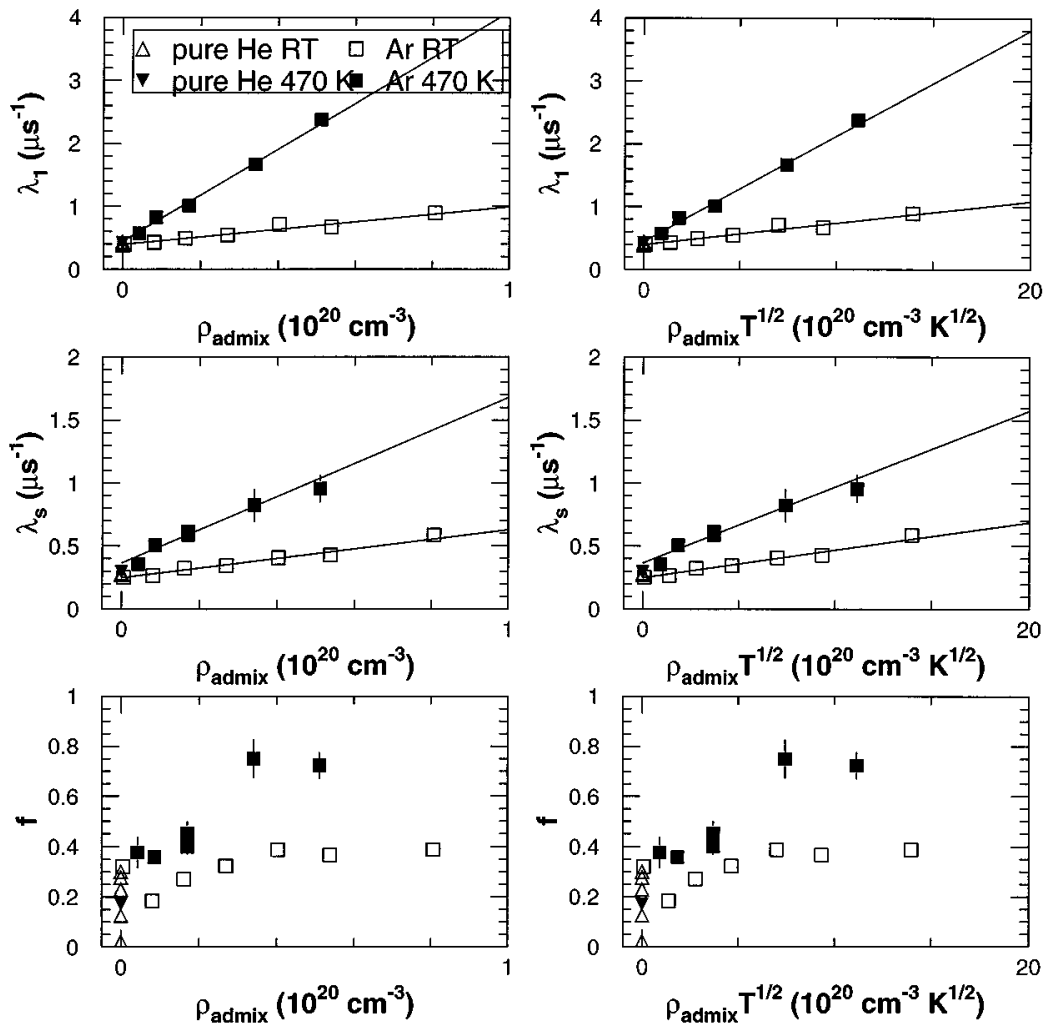

FIG. 15. Dependence of $\lambda_{1}, \lambda_{s}$, and $f$ for admixtures of $\mathrm{Ar}$ at room temperature and $470 \mathrm{~K}$ on the admixture density (left column) and on the admixture density times the square root of the temperature (right column). 


\section{ACKNOWLEDGMENTS}

We would like to thank the CERN LEAR staff for their efforts in providing us with the stable high-quality lowmomentum antiproton beam. The present work is supported by the Grant-in-Aid for Specially Promoted Scientific Re- search and International Scientific Research of the Japanese Ministry of Education, Science and Culture and the International Joint Research Project of the Japan Society for the Promotion of Science. Two of the authors (S.N.N. and E.W.) acknowledge financial support from JSPS.
[1] M. Iwasaki, S.N. Nakamura, K. Shigaki, Y. Shimizu, H. Tamura, T. Ishikawa, R.S. Hayano, E. Takada, E. Widmann, H. Outa, M. Aoki, P. Kitching, and T. Yamazaki, Phys. Rev. Lett. 67, 1246 (1991).

[2] T. Yamazaki, M. Aoki, M. Iwasaki, R.S. Hayano, T. Ishikawa, H. Outa, E. Takada, H. Tamura, and A. Sakaguchi, Phys. Rev. Lett. 63, 1590 (1989).

[3] S.N. Nakamura, M. Iwasaki, H. Outa, R.S. Hayano, Y. Watanabe, T. Nagae, T. Yamazaki, H. Tada, T. Numao, Y. Kuno, and R. Kadono, Phys. Rev. A 45, 6202 (1992).

[4] G.T. Condo, Phys. Lett. 9, 65 (1964).

[5] J.E. Russell, Phys. Rev. Lett. 23, 63 (1969); Phys. Rev. 188, 187 (1969); Phys. Rev. A 1, 721 (1970); 1, 735 (1976); 1, 742 (1970); J. Math. Phys. 12, 1906 (1971); Phys. Rev. A 6, 2488 (1972).

[6] R.S. Hayano, M. Iwasaki, and T. Yamazaki, in Perspectives of Meson Science, edited by T. Yamazaki, K. Nakai, and K. Nagamine (North-Holland, Amsterdam, 1992), p. 417.

[7] T. Yamazaki and K. Ohtsuki, Phys. Rev. A 45, 7782 (1992).

[8] R. Ahlrichs, O. Dumbrajs, H. Pilkuhn, and H.G. Schlaile, Z. Phys. A 306, 297 (1982).

[9] I. Shimamura, Phys. Rev. A 46, 3776 (1992).

[10] P.T. Greenland and R. Thürwächter, Hyperfine Interactions 76, 355 (1993).

[11] O. I. Kartavtsev (private communication).

[12] Y. Kino (private communication).

[13] K. Ohtsuki (private communication).

[14] N. Morita, K. Ohtsuki, and T. Yamazaki, Nucl. Instrum. Methods Phys. Res. Sect. A 330, 439 (1993).
[15] T. Yamazaki, E. Widmann, R.S. Hayano, M. Iwasaki, S.N. Nakamura, K. Shigaki, F.J. Hartmann, H. Daniel, T. von Egidy, P. Hofmann, Y.-S. Kim, and J. Eades, Nature 361, 238 (1993).

[16] N. Morita, M. Kamakura, T. Yamazaki, E. Widmann, H. Masuda, I. Sugai, R.S. Hayano, F.E. Maas, H.A. Torii, F.J. Hartmann, H. Daniel, T. von Egidy, B. Ketzer, W. Müller, W. Schmid, D. Horvath, and J. Eades, Phys. Rev. Lett. 72, 1180 (1994).

[17] R.S. Hayano, F.E. Maas, H.A. Torii, N. Morita, M. Kamakura, T. Yamazaki, H. Masuda, I. Sugai, F.J. Hartmann, H. Daniel, T. von Egidy, B. Ketzer, W. Müller, W. Schmid, D. Horváth, J. Eades, and E. Widmann, Phys. Rev. Lett. 73, 1485 (1994); 73, 3181 (1994).

[18] F.E. Maas, R.S. Hayano, T. Ishikawa, H. Tamura, H.A. Torii, N. Morita, T. Yamazaki, I. Sugai, K. Nakayoshi, F.J. Hartmann, H. Daniel, T. von Egidy, B. Ketzer, A. Niestroj, S. Schmid, W. Schmid, D. Horváth, J. Eades, and E. Widmann, Phys. Rev. A 52, 4266 (1995).

[19] S.N. Nakamura, R.S. Hayano, M. Iwasaki, K. Shigaki, E. Widmann, T. Yamazaki, H. Daniel, T. von Egidy, F.J. Hartmann, P. Hofmann, Y.-S. Kim, and J. Eades, Phys. Rev. A 49, 4457 (1994).

[20] E. Widmann, I. Sugai, T. Yamazaki, R. S. Hayano, M. Iwasaki, S.N. Nakamura, H. Tamura, T.M. Ito, A. Kawachi, N. Nishida, W. Higemoto, Y. Ito, N. Morita, F. J. Hartmann, H. Daniel, T. von Egidy, W. Schmid, J. Hoffmann, and J. Eades, Phys. Rev. A 51, 2870 (1995). 